\title{
Primary cilia-mediated intercellular signaling in hair follicles
}

\author{
Kazuyuki Matsushima, Mika Suematsu, Chie Mifude and Kuniyoshi Kaseda* \\ Saravio Central Institute, Saravio Cosmetics Ltd., Beppu, Japan
}

\begin{abstract}
Dermal papilla cells (DPCs) play a major role in the physiology of hair follicles. The dynamic hair growth is achieved through the sound intercellular communication. It has been demonstrated that the interactive characteristics of DPCs become more remarkable upon the spheroid formation. However, the molecular basis of the activation has remained unclear. Here we show that the novel function of sensory organelle, i.e., primary cilia, of DPCs in the intercellular interaction with other cells.

When the formation of primary cilia was inhibited, the DPC-conditioned media became less effective in the augmenting activity of the cellular proliferation of other mesenchymal stromal cells. In the same condition, it was revealed that the gene expression of FGF10 was down-regulated in DPCs. These results indicate that the primary cilia is involved in the production of the growth factor, which could in turn augment the cell growth.

Compared to monolayered cells, spheroidal DPCs showed considerably longer primary cilia. The microsphere accordingly supported the growth of the acceptor cells more efficiently than the $2 \mathrm{D}$ cultured cells. However, the proliferation efficiency became lower as the spheroidal size became larger. Interestingly, the appearance frequency of primary cilia was reduced in the larger spheroids while the length of the residual organelle kept unchanged.

Collectively, our current findings suggest that DPCs tune both the length and the appearance frequency of primary cilia, through which the cells regulate the production of signaling molecules and thereby communicate with other cells.
\end{abstract}

Abbreviations: DPC: dermal papilla cell; siRNA: small-interfering RNA; DCM: DPC-conditioned medium; siKif3a: Kif3a-siRNA

\section{Introduction}

Dermal papilla cells (DPCs) communicate with epidermal and mesenchymal-derived cells by secreting various intercellular signaling molecules, and thereby the cells play essential roles in hair growth and development $[1,2]$. The hair-inducing activity is regained by forming spheroidal micro tissues that mimic the physiological structure of dermal papillae [3]. It was demonstrated that the three-dimensional spheroidal DPCs express higher levels of intercellular signaling genes, such as wnt, bmp and $f g f$, compared to two-dimensional monolayered DPCs $[4,5]$.

A great amount of attention has been paid to the primary cilium as a signaling center in mammalian cells. It is reported that the organelle coordinates molecular signaling through Wnt-, FGF- or PDGFinvolved pathways [6]. The primary cilia of dermal cells were shown to be critical for hair follicle morphogenesis in mice: when either Kif3a or Ift 88 was knocked-out in the dermal cells, the mice showed severe lack of hair [7]. However, the direct role of the primary cilia of DPCs in intercellular signaling remains to be elucidated. In addition, the role of the organella in the hair growth cycle has not been understood.

In this study, by using a small-interfering technology and examining the spheroidal DPCs, we showed that the primary cilia of DPCs are involved in the regulation of intercellular signaling with other cells.

\section{Materials and methods}

\section{Cell culture}

Human primary DPCs from 2 Caucasian females were purchased from PromoCell (Heidelberg, Germany). The cells were routinely grown in Dulbecco's modified Eagle's medium (DMEM) supplemented with $4 \%$ fetal calf serum (FCS). 3T3-L1 fibroblasts, a type of mesenchymalderived cell line, were maintained in DMEM supplemented with $10 \%$ FCS. Fifty $\mathrm{U} / \mathrm{ml}$ penicillin and $50 \mu \mathrm{g} / \mathrm{ml}$ streptomycin were added into these media. The cells were kept in a $5 \% \mathrm{CO}_{2}$ atmosphere at $37^{\circ} \mathrm{C}$.

\section{Formation of spheroidal DPCs}

DPCs were placed on ultra-low-attachment plates (Corning Inc., New York, USA) at cellular densities of $0.5-3.0 \times 10^{4}$ cells/ well. After 3 days, the resultant spheroids were subjected to either immunofluorescent staining or the determination of the cell number (explained below).

\section{Preparation of DPC-conditioned media (DCM)}

To prepare DCM from the siRNA-treated DPCs, siRNA-lipofection was performed in the section below. DCM was collected after 24 hours of the incubation. DCMs were clarified by centrifugation at $10,000 \mathrm{xg}$ for $3 \mathrm{~min}$ before use.

\section{Determination of DPC number}

After harvesting DCM, DPCs were treated with trypsin/EDTA at

Correspondence to: Kuniyoshi Kaseda, Saravio Central Institute, Saravio Cosmetics Ltd., 1356-6 Oaza Tsurumi, Beppu, Oita 874-0840, Japan, Tel: +81(0)977-75-8575; Fax: +81-(0)977-75-8112; E-mail: kaseda@saravio.jp

Key words: dermal papilla cell; primary cilium; FGF10; spheroid; intercellular signaling; hair follicle

Received: June 05, 2016; Accepted: June 21, 2016; Published: June 24, 2016 
$37^{\circ} \mathrm{C}$. The cells were collected by centrifugation at $2,000 \mathrm{x}$ g for $1 \mathrm{~min}$, re-suspended in DMEM and treated with $500 \mu \mathrm{g} / \mathrm{ml}$ MTT. After 4 hours of the incubation, the number of the stained cells were counted under a microscope, using Image software. Finally, the cells were dissolved in dimethyl sulfoxide to determine the MTT activity as mentioned below.

\section{Small-interfering RNA (siRNA) treatment}

In order to inhibit the formation of primary cilia, siRNA-mediated silencing of the Kif3a gene was carried out according to the following procedures. DPCs $\left(3 \times 10^{4}\right.$ cells/well $)$ were lipofected with $20 \mathrm{nM}$ Kif3asiRNA oligomers (sequence 5'-AAG ACC UGA UGU GGG AGU U-3') or with a control siRNA (Allstars Negative Control siRNA, \#1027280, Qiagen, Chatsworth, CA, USA) using Oligofectamine (Invitrogen, Carlsbad, CA, USA). After the incubation for 24 hours, the medium was exchanged for fresh DMEM and the cells were further cultured for 24 hours before the fixation.

\section{Immunofluorescent staining}

DPCs (passage 6) were seeded on collagen-coated coverslips and cultured for 2 days. The cells were fixed with $4 \%$ paraformaldehyde for $30 \mathrm{~min}$ at room temperature. After washing with phosphate buffered saline ( $\mathrm{pH} 7.4$ ), primary cilia were labelled with a mouse antiacetylated $\alpha$-tubulin antibody (clone 6-11B-1; Sigma-Aldrich, St Louis, MO, USA; 1:5,000 dilution). A centrosome marker (rabbit polyclonal anti- $\gamma$-tubulin, T3559; Sigma-Aldrich, St Louis, MO, USA; 1:5,000 dilution) was used to spot the origin of primary cilia. An Alexa Fluor 555-conjugated goat anti-mouse IgG antibody (Molecular Probes, Eugene, OR, USA; 1:1,000 dilution) and an Alexa Fluor 488-conjugated goat anti-rabbit IgG antibody (Molecular Probes, Eugene, OR, USA; 1:1,000 dilution) were used as secondary antibodies. Hoechst 33342 (Dojin Kagaku, Kumamoto, Japan) was used to visualize nuclei.

\section{Measurements of the length of primary cilia}

The immunostained DPCs were examined under an Eclipse E800 microscope (Nikon, Tokyo, Japan). Images of primary cilia lying parallel to the focus plane were analyzed using ImageJ software. Primary cilia ( $n=50$ to over a hundred of two independent experiments) were examined.

\section{Proliferation assay}

DPCs and 3T3-L1 fibroblasts were used as the donor and the acceptor cells, respectively. 3T3-L1 fibroblasts $\left(1 \times 10^{3}\right.$ cells/well $)$ were cultured for 48 hours in the mixture of DCM and fresh DMEM at a 4:1 ratio. The cells were treated with $500 \mu \mathrm{g} / \mathrm{ml} \mathrm{3-(4,5-dimethylthiazol-}$ 2-yl)-2,5-diphenyltetrazolium bromide (MTT, Dojin Kagaku, Kumamoto, Japan). After 4 hours of the incubation, the cells were dissolved in dimethyl sulfoxide. The absorbance was measured at a wavelength of $570 \mathrm{~nm}$ with a reference wavelength of $650 \mathrm{~nm}$, using a Multiskan FC Microplate photometer (Thermo scientific, Waltham, MA, USA).

The signaling efficiency is defined as the proliferation activity of the acceptor cells per that of DPCs and it was calculated with the formula $\left(A_{1}-A_{0}\right) / A_{D P}$. Abbreviations: $A_{1}$, the absorbance of the acceptor cells that were cultured in DCM; $A_{0}$, the absorbance of the acceptor cells that were cultured in a control DPC-free medium; $A_{D P}$, the absorbance of donor DPCs.

\section{Real-time polymerase chain reaction (PCR)}

Total RNA extraction, cDNA synthesis and real-time PCR were performed using ReliaPrep ${ }^{\text {Tx }}$ RNA Cell Miniprep System (Promega, Madison, WI), ReverTra Ace qPCR RT Master Mix and THUNDERBIRD SYBR qPCR Mix (TOYOBO, Osaka, Japan), respectively, according to the manufacturer's instructions. The following primers were purchased from Takara (Osaka, Japan): FGF10 forward primer, 5'-GCA TGT GCG GAG CTA CAA TCA-3'; reverse primer, 5'-ACG GCA ACA ACT CCG ATT TCT AC-3'.

\section{Statistical analysis}

Data are presented as mean +/- SD. Data analysis was conducted using Kaleidagraph software (Synergy Software, Reading, PA). For primary cilia length, non-parametric statistical analysis (MannWhitney $U$ test) was performed due to the skewed nature of the data. In the other analysis, a two-tailed approach was performed.

\section{Results and discussion}

The average lengths of primary cilia of untreated and the control siRNA-treated DPCs were $2.2 \pm 1.2 \mu \mathrm{m}$ and $1.6 \pm 0.9 \mu \mathrm{m}$, respectively, which are similar to the previous observations in other mammalian cells [8]. The Kif3a-siRNA-treated DPCs showed extremely shorter primary cilia $(0.6 \pm 0.6 \mu \mathrm{m}$, Figure $1 \mathrm{C}, \mathrm{D})$. In order to investigate the role of primary cilia in intercellular signaling, DCM were added into the media of 3T3-L1 fibroblasts, and the proliferation of the acceptor cells was determined. With the siKif3a-treatment of the donner cells, the cellular proliferation of the acceptor cells was severely inhibited (Figure $1 \mathrm{E})$. The result suggests that primary cilia are involved in producing signaling factors that augment the growth of the fibroblast. Indeed, a real-time PCR analysis revealed that the gene expression of FGF10 was markedly decreased in the siKif3a-treated DPCs (Figure 1F). The growth factor is a favorite candidate that augments the proliferation of the fibroblasts and other cells [9].

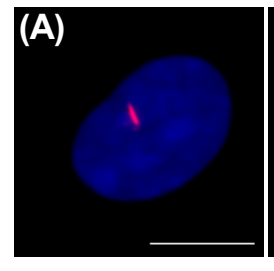

(D)

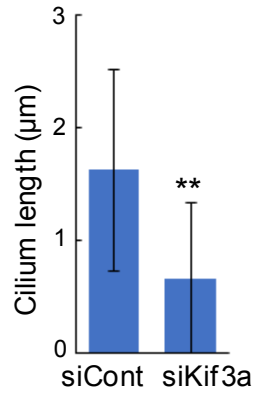

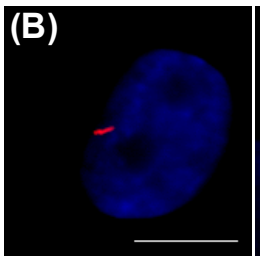

(E)

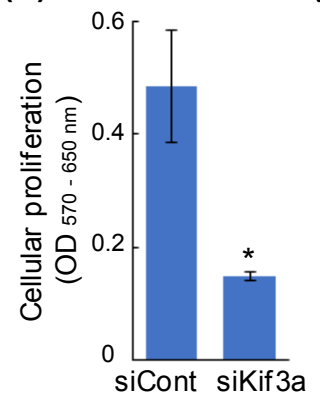

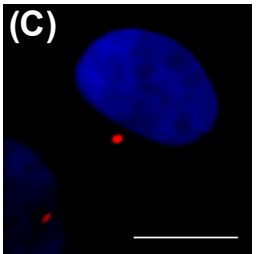

(F)

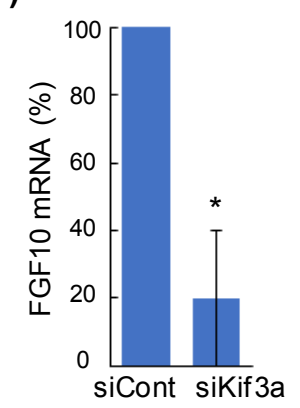

Figure 1. The regulation of intercellular signaling via primary cilia in DPCs. Primary cilia were visualized using an anti-acetylated $\alpha$-tubulin antibody (red). Nuclei were stained with Hoechst 33342 dye (blue). Scale bar $=10 \mu \mathrm{m}$. (A) Untreated DPC; (B) negative control siRNA (siCont); (C) Kif3a-siRNA (siKif3a). (D) Kif3a-knockdown shortens the primary cilia. (E) The primary cilia of DPCs regulate the proliferation of other fibroblasts. The siRNA-based DCMs were transferred into the culture of 3T3-L1 fibroblasts and the cellular proliferation of the acceptor cells were quantified after 2 days. (F) Primary cilia are involved in the gene expression of FGF10. Real-time PCR quantitation was performed in the control and the siKif3a-treated DPCs. Data are represented as the mean +/- SD. * and ** indicate $P$-values $<0.05$ and $<0.001$, respectively, compared with control. 
It is possible that spheroidal DPCs exhibit more efficient signaling transduction since DPCs acquire the hair-inducing activity upon the spheroid formation. In order to further understand the physiological role of primary cilia, the length and the appearance frequency of the organella were determined in DPC spheroids at different cellular densities. The DPC spheroids protruded significantly longer primary cilia than the monolayered DPCs over the range (Figure 2A, B). The appearance frequency of the primary cilia in DP-spheroids was higher than that in monolayered DPCs at the lower cell densities. However, the appearance frequency dramatically decreased as the cell number increased. In order to re-explore the signaling-related function of primary cilia, the proliferation activity of the acceptor 3T3-L1 fibroblasts was determined using the spheroid-derived DCM. Since the inner cells are known to be less active in spheroids [10], the signaling efficiency was calculated for both spheroidal and monolayered DPCs. As shown in Figure 2C, spheroidal DPCs showed higher signaling efficiency over the wide range of cell densities. The effect was evident at
(A)

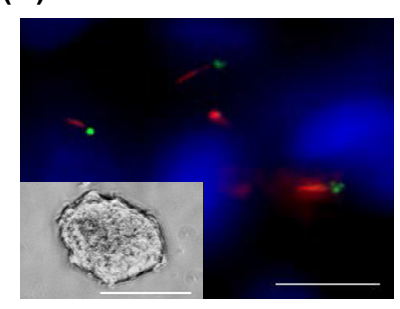

(B)

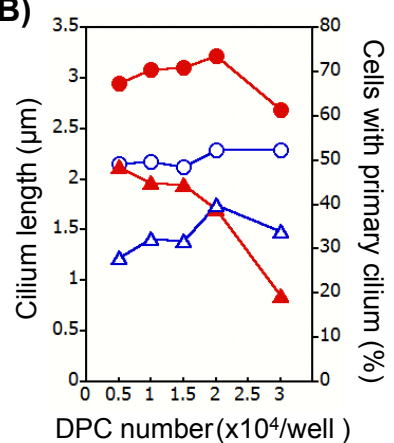

(C)

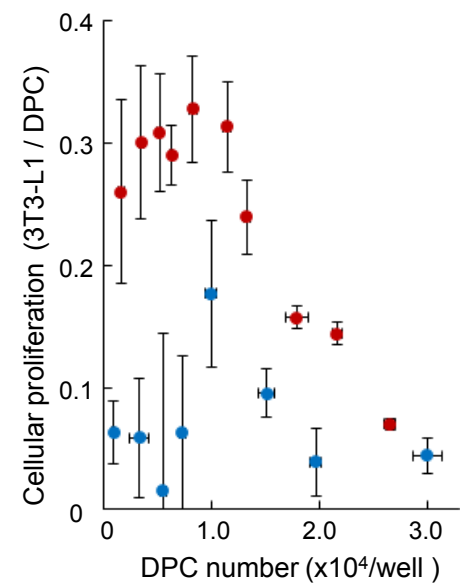

(D)

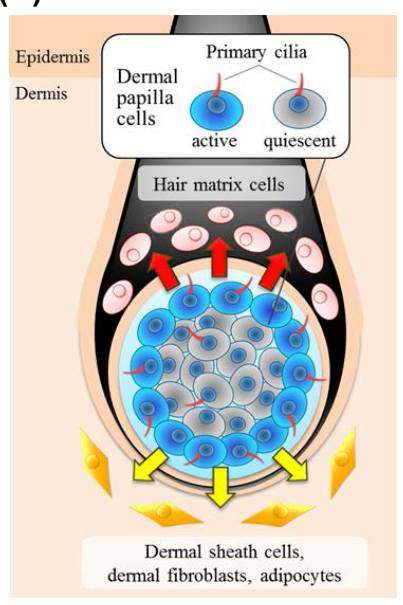

Figure 2. DPCs in spheroids show elongated primary cilia and effective signaling efficiency. (A) Immunocytochemistry of the primary cilia in spheroidal DPCs. Antiacetylated $\alpha$-tubulin (red), anti- $\gamma$-tubulin (green) antibodies and Hoechst 33342 dye (blue) were used. Scale bar $=10 \mu \mathrm{m}$. A phase-contrast image of a DPC spheroid (inset, scale bar $=100 \mu \mathrm{m}$ ). (B) The length and the appearance frequency of primary cilia. Open blue circles and closed red circles represent cilium lengths of monolayered and spheroidal DPCs, respectively. Open blue triangles and closed red triangles indicate the appearance frequencies of the primary cilia in monolayered and spheroidal DPCs, respectively. (C) DPC spheroid shows higher signaling efficiency than DPC monolayer. DCMs were prepared from either spheroidal DPCs or monolayered DPCs. 3T3-L1 fibroblasts were incubated with these conditioned media. Then, the cellular proliferations of the acceptor fibroblasts were estimated 2 days later. The proliferation activity of 3T3-L1 fibroblast per viable DPC are plotted as signaling efficiency. Red circles, DPC spheroid; blue circles, DPC monolayer. (D) A proposed model. DPCs tune the length and the appearance frequency of the primary cilia, by which the cells regulate the production of signaling factors (arrows). The outer DPCs are active, while the inner DPCs are relatively quiescent. the lower cell densities $\left(<1.0 \times 10^{4}\right.$ cells/well $)$. However, it became less evident at the higher densities, where the appearance frequency of the primary cilia became smaller in the spheroid.

We confirmed that spheroidal DPCs express higher levels of mRNA of signaling molecules such as FGF10, Wnt5a, BMP2 and FGF7 (unpublished data). These molecules are potentially involved in the effective intercellular signaling of spheroidal DPCs. Based on the current results, we suggest that the signaling efficiency of spheroidal DPCs is tuned by the regulation of the length and the appearance frequency of the primary cilia. The detailed mechanism is to be further investigated by dissecting inner and outer DPCs in different sizes of spheroids.

It is reported that photoreceptor cells release aged disc-shaped membranes from the distal ends of cilia during the process of the renewal of photoreceptor membranes [11]. In Chlamydomonas, flagella release ciliary vesicles that contain enzymes in order to hatch from the mother cell walls [12]. However, to the best of our knowledge, little is known whether or not primary cilia could mediate production and/or secretion of signaling molecules in mammalian cells. In this work, we showed that the gene expression of FGF10 decreases in the siKif3a-treated DPCs and that the DCM from the same cells exhibited the reduced proliferation activity of the acceptor cells. Therefore, we suggest that the primary cilia of DPCs are in involved in the production and/or secretion of signaling factors. In our current model, DPCs, via primary cilia, regulate the proliferation activity of surrounding cells such as dermal sheath cells, dermal fibroblasts, adipocytes and hair matrix cells (Figure 2D). We hope that our findings will contribute to developing new strategies for hair growth and hair follicle regeneration.

\section{Acknowledgements}

We would like to thank Mr. Hiroyuki Watanabe and Ms. Pravini Wickramanayake for their assistance in English language editing.

\section{Conflict of interest}

The authors declare no conflict of interest associated with this manuscript.

\section{Authors' contributions}

KK designed and supervised the research. KM, MS and CM performed the experiments. KM and KK wrote the manuscript. All authors have read and approved of the final version of the manuscript.

\section{References}

1. Sennett R, Rendl M (2012) Mesenchymal-epithelial interactions during hair follicle morphogenesis and cycling. Semin Cell Dev Biol 23: 917-927. [Crossref]

2. Festa E, Fretz J, Berry R, Schmidt B, Rodeheffer M, et al. (2011) Adipocyte lineage cells contribute to the skin stem cell niche to drive hair cycling. Cell 146: 761-771. [Crossref]

3. Osada A, Iwabuchi T, Kishimoto J, Hamazaki TS, Okochi H (2007) Long-term culture of mouse vibrissal dermal papilla cells and de novo hair follicle induction. Tissue Eng 13: 975-982. [Crossref]

4. Ohyama M, Kobayashi T, Sasaki T, Shimizu A, Amagai M (2012) Restoration of the intrinsic properties of human dermal papilla in vitro. J Cell Sci 125: 4114-4125. [Crossref]

5. Higgins CA, Chen JC, Cerise JE, Jahoda CA, Christiano AM (2013) Microenvironmental reprogramming by three-dimensional culture enables dermal papilla cells to induce de novo human hair-follicle growth. Proc Natl Acad Sci U S A 110: 19679-19688. [Crossref]

6. Zaghloul NA, Brugmann SA (2011) The emerging face of primary cilia. Genesis 49 231-246. [Crossref] 
7. Lehman JM, Laag E, Michaud EJ, Yoder BK (2009) An essential role for dermal primary cilia in hair follicle morphogenesis. J Invest Dermatol 129: 438-448. [Crossref]

8. Ou Y, Ruan Y, Cheng M, Moser JJ, Rattner JB, et al. (2009) Adenylate cyclase regulates elongation of mammalian primary cilia. Exp Cell Res 315: 2802-2817. [Crossref]

9. Igarashi M, Finch PW, Aaronson SA (1998) Characterization of recombinant human fibroblast growth factor (FGF)-10 reveals functional similarities with keratinocyte growth factor (FGF-7). J Biol Chem 273: 13230-13235. [Crossref]

10. Kunz-Schughart LA, Habbersett RC, Freyer JP (1997) Mitochondrial function in oncogene-transfected rat fibroblasts isolated from multicellular spheroids. $\mathrm{Am} \mathrm{J}$ Physiol 273: C1487-1495. [Crossref]

11. Young RW (1971) The renewal of rod and cone outer segments in the rhesus monkey. J Cell Biol 49: 303-318. [Crossref]

12. Wood CR, Huang K, Diener DR, Rosenbaum JL (2013) The cilium secretes bioactive ectosomes. Curr Biol 23: 906-911. [Crossref]

Copyright: $\odot 2016$ Matsushima K. This is an open-access article distributed under the terms of the Creative Commons Attribution License, which permits unrestricted use, distribution, and reproduction in any medium, provided the original author and source are credited. 